\section{Seeing CNV sooner}

\section{By Michael J. Haas, Senior Writer}

New research by a multinational team suggests that noninvasive imaging of CC chemokine receptor 3 could help detect wet age-related macular degeneration before retinal damage occurs. ${ }^{1}$ This would allow earlier therapeutic intervention, which is important because retinal damage is not always reversible.

In addition, the researchers think $\mathrm{CC}$ chemokine receptor 3 (CCR3) inhibitors could be combined with VEGF-A inhibitors to treat the disease- even if the former are unlikely to displace the latter.

The wet form of AMD begins with choroidal neovascularization (CNV) - the abnormal growth of blood vessels in the choroid layer behind the retina. Eventually, these vessels damage the retina and can cause loss of vision and retinal detachment.

Fluorescein angiography, the standard technique for diagnosing $\mathrm{AMD}$, can only detect $\mathrm{CNV}$ after retinal damage has begun. "The major hurdle to preventing vision loss is the detection of abnormal vasculature before it damages the vision," said Jayakrishna Ambati, professor of physiology and vice chair of ophthalmology and visual sciences at the University of Kentucky.

In 2006, Ambati and colleagues reported that immune system activation plays a role in the earliest stages of aberrant angiogenesis in the eye. ${ }^{2}$ Since then, they have come to focus on chemokines because of the role those molecules have in immune system signaling.

Now, in a paper published in Nature, another team led by Ambati reported that choroidal endothelial cells from human AMD patients expressed CCR3 and its ligands, whereas choroidal cells from nonAMD patients did not. The ligands-eotaxin-1 (CCL11), eotaxin-2 (CCL24) and eotaxin-3 (CCL26) -stimulated the proliferation of human choroidal endothelial cells in vitro, whereas CCR3 inhibition prevented CNV in a mouse model of laser-induced eye injury.

Next, the researchers showed that noninvasive imaging with antiCCR3 antibody fragments conjugated to quantum dots detected neovasculature at earlier stages of growth than fluorescein angiography in a mouse model of spontaneous CNV.

In addition to the University of Kentucky, the Nature team included scientists from the Nagoya City University Graduate School of Medical Sciences, Oregon Health \& Science University, the University of Cincinnati, the University of Lubeck, The University of North Carolina at Chapel Hill, The University of Utah and the Veterans Affairs Salt Lake City Health Care System.
"The imaging technique is intriguing and interesting because there are no methods to detect pre-wet AMD patients," said Pascal Deschatelets, $\mathrm{COO}$ and cofounder of Potentia Pharmaceuticals Inc. "If you could detect and then treat the patient before blood vessel leakage damages the retina-that would be useful."

"CCR3 imaging might be used to detect early CNV in patients with long-standing dry AMD" before it converts to wet AMD, said Ryo Kubota, chairman, president and CEO of Acucela Inc.

Acucela and partner Otsuka Pharmaceutical Co. Ltd. recently completed enrollment for a Phase I trial of ACU-4429, a nonretinoid visual cycle modulator, to treat dry AMD. Kubota said the partners plan to take the compound into Phase Ib and IIa trials this year.

Mark Keating, head of the ophthalmological disease area at Novartis AG's Novartis Institutes for Biomedical Research, said early detection of CNV could be beneficial because current therapies do not reverse all retinal damage. But early diagnosis is useful "only if you have something to prevent $\mathrm{CNV}$ at this stage," he said. "We don't know yet whether Lucentis would work because we aren't currently able to diagnose the disease at such an early stage."

Lucentis ranibizumab, from Roche's Genentech Inc. unit and Novartis, targets VEGF and is the major drug indicated for AMD.

Keating noted that only $30-40 \%$ of patients who respond to Lucentis experience significant improvements in visual acuity. "The rest simply don't get worse," he said. "It might be because too much damage has been done to reverse it, or it might be because they need another therapy."

"One of the current limitations of VEGF-A inhibitor therapy is that there is no end to treatment until a patient fails to respond," said Tom Hohman, VP of retinal pharmaceutical development at Alcon Inc. "We need new treatments that could overcome this limitation" by reversing $\mathrm{CNV}$ and curing the disease.

\section{Look beyond the surface}

Ambati's team is now working to optimize the in vivo CCR3 imaging technique without using quantum dots.

"The biocompatibility of these dots is questionable because they contain cadmium, selenium and other metals that could be toxic," he said. "So one thing we're doing to move forward is switching to an organic near-infrared dye" conjugated to the anti-CCR3 antibody fragments. "We think this should be more biocompatible and less toxic than quantum dots."

A key question is whether the findings in mice will translate to humans because no mouse model of CNV is representative of the human disease.

As an example, Deschatelets noted that the laser injury-induced model is widely used but does not trigger the immune system response observed in human AMD. In addition, he said the model of spontaneous CNV that Ambati's research group used for the imaging studies is 


\section{TARGETS \& MECHANISMS}

somewhat controversial. "I think this model does indeed work, but other researchers have not been able to reproduce it," Deschatelets said.

In 2003 Ambati's group described two mouse models of spontaneous CNV: a chemokine C$\mathrm{C}$ motif ligand $2(\mathrm{Ccl} 2)$ knockout and a CC chemokine receptor 2 (Ccr2) knockout. ${ }^{3}$ The model of spontaneous knockout used in the Nature paper was a double Ccl2/Ccr2 knockout.

Novartis has worked with Ambati's Ccl2 knockout model, "but we haven't had much luck for reasons that aren't clear," Keating said. "This model may be valuable, but its value is less clear than the laser model because it has been studied less" and has not yet been the basis for any IND-enabling studies.

Ambati told SciBX that other researchers have confirmed the pathology and phenotype of all three of his group's models.

Without a good model to aid AMD drug development, "you have to go blind in the field," said Deschatelets. Indeed, Potentia's POT-4, a synthetic peptide complement 3 (C3) inhibitor, "went straight into humans without going through preclinical animal models" of efficacy, he told SciBX. The company's preclinical safety and toxicology studies were done in rabbits and monkeys.

Potentia plans to begin a Phase II trial of POT-4 to treat AMD this year.

Mouse models aside, Alcon's Hohman wanted to know whether CCR3-based imaging "would be a better predictor of CNV growth and disease progression in humans than fluorescein angiography or known risk factors" for AMD.

"Such a comparison would require a fairly extensive study to assess how predictive CCR3 is," and would have to be much larger than the limited human sample size described in the Nature paper, added Glenn Noronha, project head of retinal drug development at Alcon.

Alcon has AL-8309B, a serotonin $\left(5-\mathrm{HT}_{1 \mathrm{~A}}\right)$ receptor $\left(\mathrm{HTR}_{1 \mathrm{~A}}\right)$ agonist, in a Phase III trial to treat geographic atrophy (GA) secondary to dry AMD. The company expects to complete the trial in 2012. Last year, Alcon discontinued development of a modified steroid, Retaane anecortave acetate, to treat AMD. Retaane is approved to treat the indication in Australia and several Latin American countries.

\section{Safety on target}

In addition to the use of CCR3 in imaging, Ambati thinks that CCR3 antagonists could become part of a therapeutic cocktail with marketed VEGF inhibitors. "We found that CCR3 and VEGF-A operate by distinctly different angiogenic pathways," suggesting that combining inhibitors could have additive effects, he said.

Ambati thinks a CCR3/VEGF cocktail "would theoretically reduce the needed amount of both agents, and thus reduce any potential risks associated with anti-VEGF-A therapy."

Such risks include retinal damage in mice $e^{4,5}$ and mild photoreceptor damage in humans. ${ }^{6,7}$ The label for Lucentis includes warnings of intraocular inflammation, retinal detachment and increased intraocular pressure.

Kubota noted that CCR3 inhibition does not appear to cause these effects in mice, which he said suggests that CCR3 inhibitors might also be safer in humans.

Keating countered that it was too early to conclude whether CCR3 inhibition would be safer than Lucentis. Given that drug's track record, "it would be hard - though not impossible-to be safer," he said.

"Almost all wet AMD drug candidates are looked at as add-ons to Lucentis, because its results have been so dramatic," Keating added. "It's hard to imagine treating AMD without Lucentis."

Alternately, Deschatelets suggested that CCR3 inhibitors could be used as salvage therapy in patients who don't respond to VEGF blockers because "about $10 \%$ of patients are nonresponders to Lucentis."

Ambati said the team would like to develop anti-CCR3 therapies to treat CNV but would require a commercial partner to do so.

Keating said Novartis will "definitely follow up" on the results published in Nature. "We've done a little work with CCR3. This paper has raised this molecule in our awareness, and the first thing we would do is try to reproduce these results."

The University of Kentucky has patented the findings, and Ambati said they are available for licensing.

Haas, M.J. SciBX 2(25); doi:10.1038/scibx.2009.995

Published online June 25, 2009

\section{REFERENCES}

1. Takeda, A. et al. Nature; published online June 14, 2009; doi:10.1038/nature08151

Contact: Jayakrishna Ambati, University of Kentucky, Lexington, Ky. e-mail: jamba2@email.uky.edu

2. Nozaki, M. et al. Proc. Natl. Acad. Sci. USA 103, 2328-2333 (2006)

3. Ambati, J. et al. Nat. Med. 9, 1390-1397 (2003)

4. Nishijima, K. et al. Am. J. Pathol. 171, 53-67 (2007)

5. Saint-Geniez, M. et al. PLoS One 3, e3554; published online

Nov. 3, 2008; doi:10.1371/journal.pone.0003554

6. Sayanagi, K. et al. Br. J. Ophthalmol. 93, 622-626 (2009)

7. Yodoi, Y. et al. Am. J. Ophthalmol. 147, 816-824 (2009)

\section{COMPANIES AND INSTITUTIONS MENTIONED}

Acucela Inc., Bothell, Wash.

Alcon Inc. (NYSE:ACL), Hunenberg, Switzerland

Genentech Inc., South San Francisco, Calif.

Nagoya City University Graduate School of Medical Sciences, Nagoya, Japan

Novartis AG (NYSE:NVS; SIX:NOVN), Basel, Switzerland

Oregon Health \& Science University, Portland, Ore.

Otsuka Pharmaceutical Co. Ltd. (Tokyo:4768), Tokyo, Japan

Potentia Pharmaceuticals Inc., Louisville, Ky.

Roche (SIX:ROG), Basel, Switzerland

University of Cincinnati, Cincinnati, Ohio

University of Kentucky, Lexington, Ky.

University of Lubeck, Lubeck, Germany

The University of North Carolina at Chapel Hill, Chapel Hill, N.C.

The University of Utah, Salt Lake City, Utah

Veterans Affairs Salt Lake City Health Care System, Salt Lake City, Utah 